\title{
Congenital Ventricular Aneurysm as an Unexpected Complication of Monomorphic Premature Ventricular Contractions
}

\author{
Shusuke Yagi ${ }^{1}$, Masashi Akaike ${ }^{1}$, Mitsunori Fujimura ${ }^{2}$, Takehiko Kimura ${ }^{3}$, \\ Takeshi Nishiuchi $^{3}$, Takashi Iwase ${ }^{1}$, Ken-ichi Aihara ${ }^{4}$, Sumiko Yoshida ${ }^{4}$, \\ Yuka Sumitomo-Ueda ${ }^{4}$, Kenya Kusunose ${ }^{1}$, Toshiyuki Niki ${ }^{1}$, Koji Yamaguchi ${ }^{1}$, \\ Kunihiko Koshiba ${ }^{1}$, Yoichiro Hirata ${ }^{1}$, Munkhbaatar Dagvasumberel ${ }^{1}$, Yoshio Taketani ${ }^{1}$, \\ Noriko Tomita ${ }^{1}$, Hirotsugu Yamada ${ }^{1}$, Takeshi Soeki ${ }^{1}$, Tetsuzo Wakatsuki ${ }^{1}$, \\ Toshio Matsumoto ${ }^{4}$ and Masataka Sata ${ }^{1}$
}

\begin{abstract}
Congenital ventricular diverticulum (CVD) in adults is a rare cardiac malformation, which includes fibrous type congenital ventricular aneurysm (CVA). CVA is often clinically asymptomatic and shows no abnormality in the electrocardiogram or chest X-ray. However, some cases of sudden death resulting from ventricular tachycardia, cardiac embolism or ventricular rupture have been reported. Therefore, physicians should perform further cardiac imaging studies to detect a CVA if ventricular arrhythmia originating from the left ventricle is observed. Here, we report two successfully followed cases of CVA which were diagnosed from premature ventricular contractions.
\end{abstract}

Key words: congenital ventricular aneurysm, congenital ventricular diverticulum, ventricular arrythmia

(Inter Med 49: 907-912, 2010)

(DOI: 10.2169/internalmedicine.49.3008)

\section{Introduction}

Congenital ventricular outpouchings, which include congenital ventricular diverticulum (CVD) and congenital ventricular aneurysm (CVA), are rare cardiac malformations consisting of a localized protrusion from the free wall of the left ventricle $(1,2)$. CVD comprises both the muscular type and fibrous type, and even the fibrous type can be called CVA. The myocardial layer of the fibrous type (CVA), which shows no cardiac contraction of the aneurysm, is replaced by fibrous tissue to some extent compared to that of the muscular type (CVD), which shows preserved cardiac contraction of the aneyrysm (3). This uneven fibrotic lesion in CVA might be the cause of ventricular arrhythmia. CVA is often clinically asymptomatic and shows no abnormality in the electrocardiogram or chest X-ray. However, some cases of sudden death or critical events of ventricular tachycardia $(3,4)$, cardiac embolism $(5,6)$ or ventricular rupture (7) have been reported. Therefore, the diagnosis of CVA and evaluation of cardiac event risk are very important. Physicians should perform further cardiac imaging studies to detect a CVD or CVA if ventricular arrhythmia originating from the left ventricle is observed. Here, we report two successfully followed cases of CVA which were diagnosed from premature ventricular contractions.

\footnotetext{
${ }^{1}$ Department of Cardiovascular Medicine, The University of Tokushima Graduate School of Health Biosciences, Tokushima, ${ }^{2}$ Takamatsu Municipal Hospital, Takamatsu, ${ }^{3}$ Kawashima Cardiovascular Clinic, Tokushima and ${ }^{4}$ Department of Medicine and Bioregulatory Sciences, The University of Tokushima Graduate School of Health Biosciences, Tokushima

Received for publication October 12, 2009; Accepted for publication January 21, 2010

Correspondence to Dr. Shusuke Yagi, syagi@ clin.med.tokushima-u.ac.jp
} 


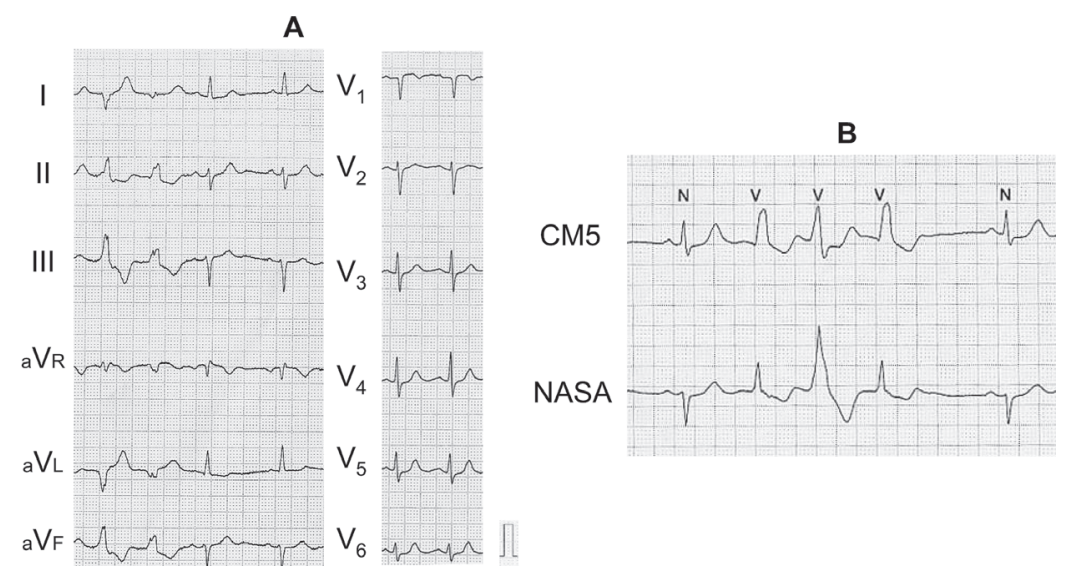

Figure 1. A: Electrocardiographic findings (Case 1). Electrocardiography revealed no significant ischemic change, but premature ventricular contractions of downward axis in limb leads were seen. B: Holter electrocardiography findings (Case 1). Triplets of premature ventricular contraction were observed. The upper lead indicates CM5 corresponding to V5 and the lower lead indicates NASA corresponding to $\mathrm{V} 2$.

A

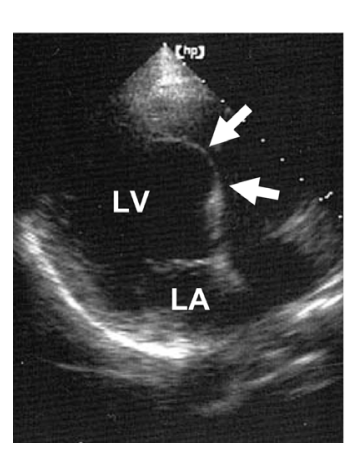

a

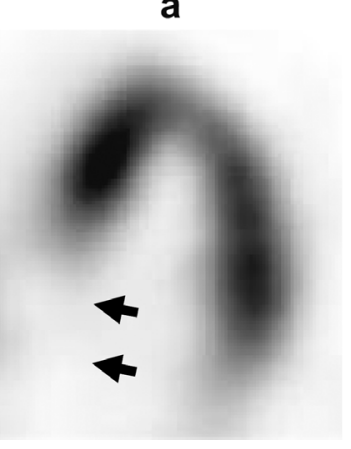

B

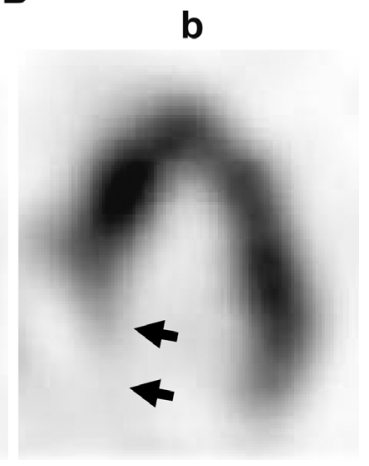

Figure 2. A: Echocardiographic findings (Case 1). Transthoracic echocardiography of left ventricle revealed aneurysmal change in the anterorseptal wall and thinning of the wall (arrow). (LV: left ventricle, LA: left atrium). B: ${ }^{201}$ Thallium exercise stress scintigraphy (Case 1). (a; early image, b; delayed image). Horizontal long-axis view of ${ }^{201}$ thallium exercise stress scintigraphy showed a defective image in the left ventricular basal anteroseptal wall (arrow).

\section{Case Report}

\section{Case 1}

In February 2002, a 47-year-old woman noticed palpitations, mainly on exertion, and was examined at our clinic in March of the same year.

The electrocardiography (ECG) revealed no significant ischemic change, but sporadic premature ventricular contractions (PVCs) of the downward axis in the limb leads were seen (Fig. 1A). The Holter ECG revealed triplet right bundle branch block pattern PVCs which originated from the anterior wall of the left ventricle at a rate of 5 per day (Fig. 1B). Echocardiography revealed thinning of the anteroseptal wall without mural thrombus and aneurysmal motion in the systolic phase at the same site (Fig. 2A). The thallium-201 $\left({ }^{201} \mathrm{Tl}\right)$ exercise stress scintigraphy showed a defective image from the base to the center of the septum of the anterior ventricular wall with no redistribution (Fig. 2B a, b). The coronary angiography showed no abnormal findings, and left ventriculography revealed aneurysmal motion in the anteroseptal wall in the basal part and preserved ejection fraction of $55 \%$ (Figs. 3A, 3B). The BNP level was slightly increased to $63.7 \mathrm{pg} / \mathrm{mL}$. She had no ocular or skin lesions of sarcoidosis clinically. Computed tomography (CT) of the chest showed no hilar or mediastinal lymphoadenopathy or lesions and no abnormality of lung fields, and gallium-67 scintigraphy showed no abnormal accumulation. Moreover, serum levels of gamma globulin, angiotensin-converting enzyme and lysozyme were not increased. The biopsy specimens obtained from three locations in the right ventricular side of the interventricular septum showed no abnormal findings. The signal-averaged electrocardiography showed no ventricular late potentials. From these findings, CVA complicated with PVCs was diagnosed. The PVCs and her symptoms disappeared after treatment with atenolol at a dose of $12.5 \mathrm{mg}$. She was observed as an 
A

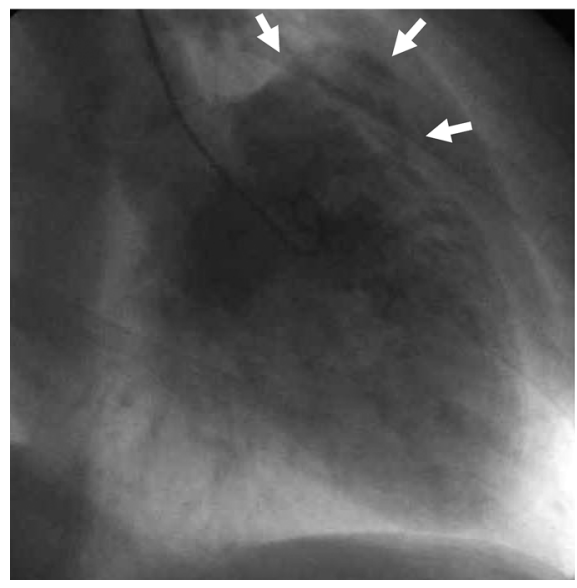

B

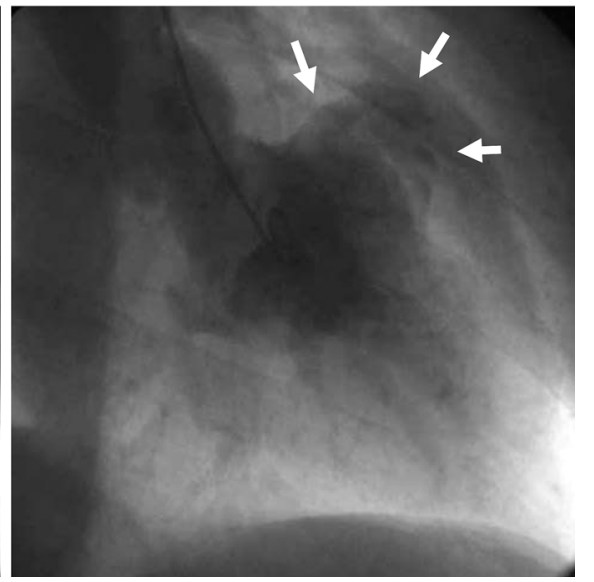

Figure 3. Left ventriculography (Case 1). (A; end-diastole, B; end-systole). Left ventriculography in 30-degree right anterior oblique projection revealed a ventricular aneurysm in the anteroseptal wall (arrow).

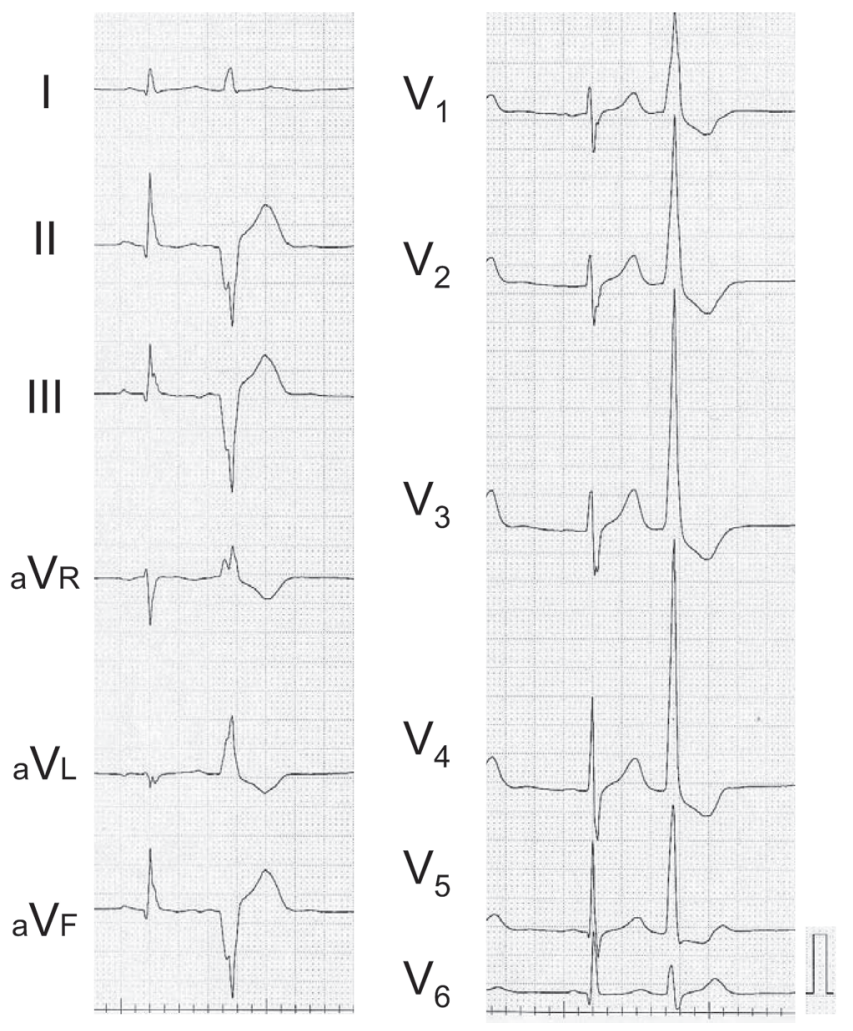

Figure 4. Electrocardiographic findings (Case 2). Electrocardiography revealed premature ventricular contractions of right bundle branch block pattern with upward axis deviation.

outpatient, and 7 years after the diagnosis she did not exhibit enlargement of the aneurismal formation or cardiovascular complications.

\section{Case 2}

In August 2004, a 77-year-old man was admitted to the surgical department of our hospital for repair of an inguinal hernia. Although he had never noticed any chest pain, he had become aware of palpitations 5 years earlier. The ECG on admission revealed PVCs of right bundle branch block pattern with upward axis deviation. The PVCs might have originated from the posterior wall of the left ventricle (Fig. 4). Due to the many episodes of PVCs, the patient was referred to our department for presurgical assessment. The Holter ECG revealed monomorphic single PVCs at a rate of about 5,000 per day. The echocardiography revealed outpouching of an aneurysm in the base of the posterior wall and wall thinning at the same site (Fig. 5A). There was no thrombus in the aneurysm. The enhanced CT showed a similar finding as the echocardiography (Fig. 5B). The ${ }^{201} \mathrm{Tl}$ dipyridamole stress scintigraphy showed defective uptake in the posterior wall and no redistribution (Fig. 5C a, b). The coronary angiography showed no abnormal findings, and left ventriculography revealed aneurysmal motion of the posterior wall in the systolic phase as well as paradoxical flow to the aneurysm in the diastolic period (Fig. 6). The left ventricular ejection fraction was mildly decreased to $54 \%$. The BNP level was slightly increased to $71.7 \mathrm{pg} / \mathrm{mL}$. As in Case 1, there were no clinical findings of cardiac sarcoidosis. Therefore, CVA complicated with PVCs was diagnosed. Surgery for the inguinal hernia was completed uneventfully. He was discharged and followed at a local hospital. Five years after the diagnosis he did not exhibit cardiovascular complications.

\section{Discussion}

CVD is a rare congenital anomaly which is an outpouching of the ventricular wall. In the adult population, the prevalence is reported to be about $0.4 \%$ in an autopsy series of patients with cardiac death and about $0.26 \%$ in unselected patients who underwent cardiac catheterization $(8,9)$. CVD is suggested to be triggered by a congenital weakness of the left ventricular free wall, although the etiology has not been elucidated. From the pathological point of view, the fibrous 

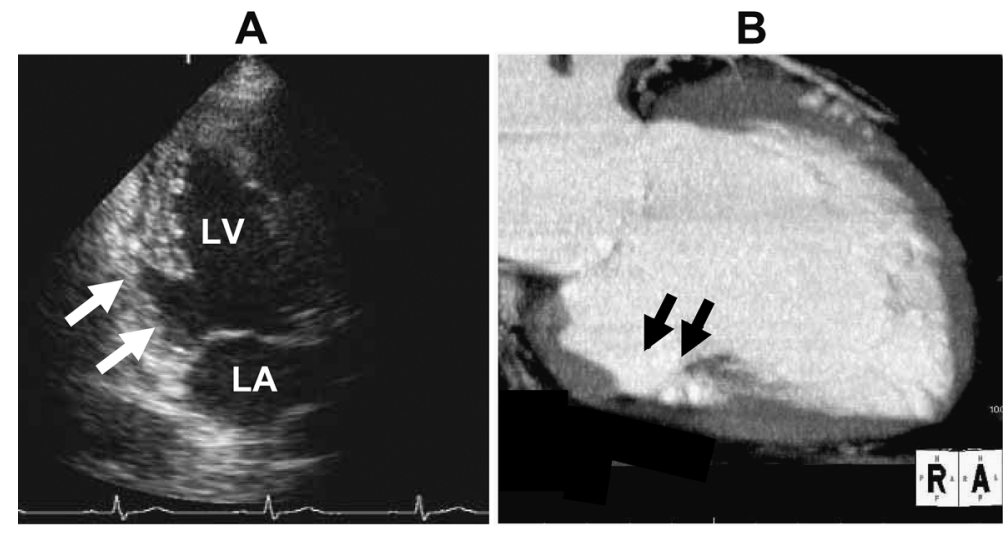

C

a

b

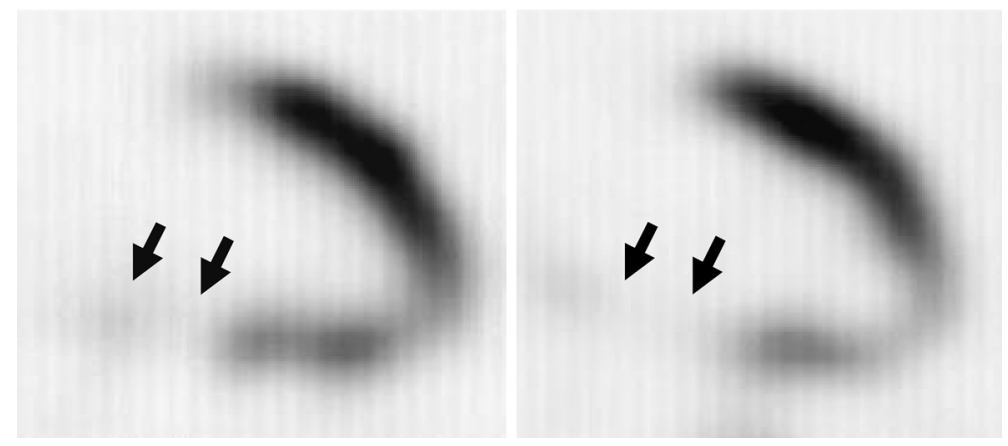

Figure 5. A: Echocardiographic findings (Case 2). Transthoracic echocardiography revealed left ventricular aneurysmal change in the base of the posterior wall with thinning of the wall (arrow). (LV; left ventricle, LA; left atrium). B: Enhanced computed tomographic findings (Case 2). Enhanced computed tomography showed left ventricular aneurysmal change in the basal posterior wall (arrow). C: Dipyridamole stress ${ }^{201}$ thallium scintigraphy (Case 2). (a; early image, b; delayed image). Vertical long-axis view of ${ }^{201}$ thallium dipyridamole stress scintigraphy showed defective uptake in the left ventricular basal posterior wall (arrow).

A

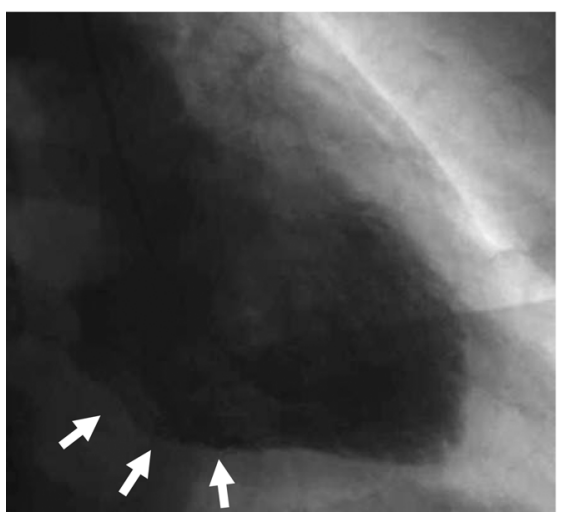

B

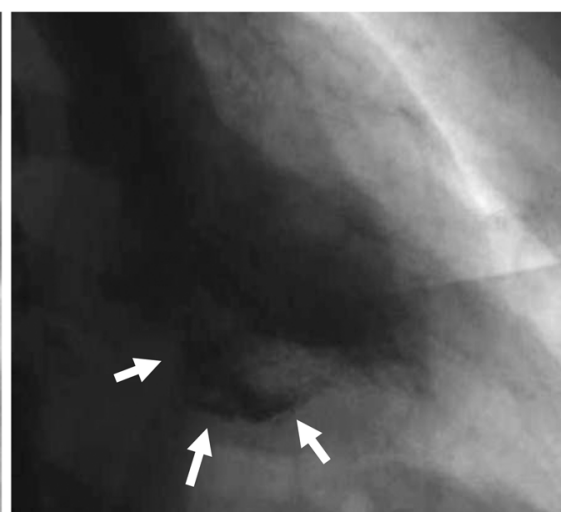

Figure 6. Left ventriculography (Case 2). (A; end-diastole, B; end-systole). Left ventriculography in 30-degree right anterior oblique projection revealed a left ventricular aneurysm in the basal posterior wall (arrow).

type of CVD is called CVA. The structure of the three myocardial layers is preserved in CVA. However, the myocardium is replaced by fibrous tissue to some extent, which might be responsible for ventricular arrhythmia. In these two cases, the ${ }^{201} \mathrm{Tl}$ scintigraphy showed almost no accumulation at the site of the aneurysm. These findings suggested that myocardial tissue was replaced by fibrous tissue.

Because CVD including CVA is often asymptomatic and is usually found coincidentally during diagnostic procedures performed for other diseases, the CVD was diagnosed in elderly years (64 \pm 9 years) (10). The natural history of a CVD is not completely understood, but most patients remain 
asymptomatic and without complications throughout their lifetime (11). However, some cases of sudden death resulting from ventricular tachycardia or ventricular rupture had an extremely bad prognosis, with only $30 \%$ of patients alive at 4 years (12). Therefore, it is very important to diagnose CVD and to evaluate cardiac event risk.

Echocardiography is a readily available and useful tool for the screening and diagnosis of CVD. It allows accurate detection of CVD and assessment of its morphology, location, possible thrombosis and associated congenital cardiac disorders. The important diagnostic characteristics of CVD are a narrow neck, normal ejection fraction, systolic flow pattern from the diverticulum to the ventricle, and small size and circular shape (13). Magnetic resonance imaging with delayed contrast enhancement has been used recently, because it can detect areas of left ventricular fibrosis sensitively and distinguish fibrous type from muscular type $(14,15)$. Cardiac CT and left ventriculography are important tools to demonstrate anatomical changes, and scintigraphic imaging with ${ }^{201} \mathrm{Tl}$ is also useful to evaluate the degree of fibrous change.

Therapeutic recommendations are difficult to derive since only case reports or small series have been published in the literature, and larger trials with longer observation periods are lacking. It has been reported that the location of aneurysms in cases of sudden death involved inferior or posterior wall segments (11). However, the association between location of the aneurysm and clinical course has not been fully elucidated. Some CVD patients exhibit ventricular arrhythmia including occasional PVCs to sudden cardiac death. The majority of ventricular arrhythmias arise from reentry circuits originating in the CVD in patients with symptomatic ventricular arrhythmia (11). CVD might be an arrhythmogenic substrate in the left ventricle (16-18). Patients with symptomatic ventricular arrhythmia might require electrophysiological study to determine the substrate of arrythmia or to evaluate the risk for cardiac sudden death. Radiofrequency catheter ablation or implantation of a cardiac defibrillator might also be needed depending on the risk of sudden death.

A nonsurgical strategy with careful follow-up has been chosen in many case reports as in our two cases. This may be supported by the report in a series of 16 patients with CVA. In this series, an uneventful course was demonstrated in $94 \%$ of the patients over a period of up to 127 months (mean period of 61 months). Although the cardiac event rate was approximately $1.2 \%$ per year, no cardiac death was seen during the follow-up period (16). Therefore, observational follow-up might be recommended in patients with a low risk for cardiac sudden death.

The present two cases had no history of symptoms such as syncope or faintness associated with ventricular tachycardia and no thromboembolic event before attending our hospital. Their left ventriculography showed preserved global cardiac systolic function. The former patient had atenololresponsive triplets with negative late potential. The latter patient had only single PVCs. Therefore, we evaluated that the risk of cardiac sudden death due to fatal ventricular arrhythmia was low and chose observational follow-up. They had no cardiac event on observational follow-up for 7 years and 5 years after the diagnosis of CVA, respectively. Physicians should perform additional cardiac imaging studies to detect a CVA if ventricular arrhythmia originating from the left ventricle is observed.

\section{References}

1. Hamaoka K, Onaka M, Tanaka T, Onouchi Z. Congenital ventricular aneurysm and diverticulum in children. Pediatr Cardiol 8: 169-175, 1987.

2. Cavalle-Garrido T, Cloutier A, Harder J, Boutin C, Smallhorn JF. Evolution of fetal ventricular aneurysms and diverticula of the heart: an echocardiographic study. Am J Perinatol 14: 393-400, 1997.

3. Shen EN, Fukuyama O, Herre JM, Yee E, Scheinman MM. Ventricular tachycardia with congenital ventricular diverticulum. Chest 100: 283-285, 1991.

4. Yamashiro S, Kuniyoshi Y, Miyagi K, Uezu T, Arakaki K, Koja $\mathrm{K}$. Two cases of ventricular tachycardia with congenital left ventricular malformation in an adult. Ann Thorac Cardiovasc Surg 10: 42-46, 2004.

5. Bell WE, Ehmke DA. Diverticulum of the left ventricle in a child with fatal cerebral embolization. South Med J 64: 537-540, 1971.

6. Chesler E, Tucker RB, Barlow JB. Subvalvular and apical left ventricular aneurysms in the Bantu as a source of systemic emboli. Circulation 35: 1156-1162, 1967.

7. Meyersohn J, Schiffer J. Rupture of a congenital aneurysm of the left ventricular apex. Chest 63: 838-840, 1973.

8. Skapinker S. Diverticulum of the left ventricle of the heart; review of the literature and report of a successful removal of the diverticulum. AMA Arch Surg 63: 629-634, 1951.

9. Walton-Shirley M, Smith SM, Talley JD. Left ventricular diver- ticulum: case report and review of the literature. Cathet Cardiovasc Diagn 26: 31-33, 1992.

10. Ohlow MA, Secknus MA, Geller JC, von Korn H, Lauer B. Prevalence and outcome of congenital left ventricular aneurysms and diverticula in an adult population. Cardiology 112: 287-293, 2009.

11. Cianciulli TF, Del Carmen Gonzalez Colaso P, Saccheri MC, et al. Left ventricular diverticulum, a rare echocardiographic finding: two adult patients and review of the literature. Cardiol J 16: 7681, 2009.

12. Marijon E, Ou P, Fermont L, et al. Diagnosis and outcome in congenital ventricular diverticulum and aneurysm. J Thorac Cardiovasc Surg 131: 433-437, 2006.

13. Teske DW, McGovern JJ, Allen HD. Congenital fibrous left ventricular diverticulum. Am Heart J 126: 1233-1235, 1993.

14. Awad SM, Patel AS, Polimenakos A, Braun R, Abdulla RI. Left ventricular accessory chamber: a case report and review of the literature. Pediatr Cardiol 30: 1022-1025, 2009.

15. McMahon CJ, Moniotte S, Powell AJ, del Nido PJ, Geva T. Usefulness of magnetic resonance imaging evaluation of congenital left ventricular aneurysms. Am J Cardiol 100: 310-315, 2007.

16. Mayer K, Candinas R, Radounislis C, Jenni R. Congenital left ventricular aneurysms and diverticula: clinical findings, diagnosis and course. Schweiz Med Wochenschr 129: 1249-1256, 1999 (in German). 
Inter Med 49: 907-912, 2010 DOI: 10.2169/internalmedicine.49.3008

17. Ouyang F, Antz M, Deger FT, et al. An underrecognized subepicardial reentrant ventricular tachycardia attributable to left ventricular aneurysm in patients with normal coronary arteriograms. Circulation 107: 2702-2709, 2003.
18. Fellows CL, Bardy GH, Ivey TD, Werner JA, Draheim JJ, Greene HL. Ventricular dysrhythmias associated with congenital left ventricular aneurysms. Am J Cardiol 57: 997-999, 1986.

(C) 2010 The Japanese Society of Internal Medicine http://www.naika.or.jp/imindex.html 